\title{
Corrigendum: Repeatability and Reproducibility of in-vivo Brain Temperature Measurements
}

\author{
Ayushe A. Sharma ${ }^{1,2,3 *}$, Rodolphe Nenert ${ }^{3,4}$, Christina Mueller ${ }^{1}$, Andrew A. Maudsley ${ }^{5}$, \\ Jarred W. Younger ${ }^{1}$ and Jerzy P. Szaflarski ${ }^{2,3,4,6}$ \\ ${ }^{1}$ Department of Psychology, University of Alabama at Birmingham (UAB), Birmingham, AL, United States, ${ }^{2}$ Department of \\ Neurobiology, University of Alabama at Birmingham (UAB), Birmingham, AL, United States, ${ }^{3}$ University of Alabama at \\ Birmingham Epilepsy Center (UABEC), Birmingham, AL, United States, ${ }^{4}$ Department of Neurology, University of Alabama at \\ Birmingham (UAB), Birmingham, AL, United States, ${ }^{5}$ Department of Radiology, Miller School of Medicine, University of \\ Miami, Miami, FL, United States, ${ }^{6}$ Department of Neurosurgery, University of Alabama at Birmingham (UAB), Birmingham, \\ AL, United States
}

Keywords: MRS, brain temperature, MR thermometry, neuroinflammation, neuroimaging

\section{A Corrigendum on}

\section{OPEN ACCESS}

Edited and reviewed by: Dajiang Zhu,

University of Texas at Arlington, United States

*Correspondence: Ayushe A. Sharma sharma87@uab.edu

Specialty section: This article was submitted to Brain Imaging and Stimulation, a section of the journal Frontiers in Human Neuroscience

Received: 21 September 2021 Accepted: 04 November 2021 Published: 26 November 2021

Citation:

Sharma AA, Nenert R, Mueller $C$

Maudsley $A A$, Younger JW and Szaflarski JP (2021) Corrigendum: Repeatability and Reproducibility of in-vivo Brain Temperature Measurements.

Front. Hum. Neurosci. 15:780797. doi: 10.3389/fnhum.2021.780797
Repeatability and Reproducibility of in-vivo Brain Temperature Measurements

by Sharma, A. A., Nenert, R., Mueller, C., Maudsley, A. A., Younger, J. W., and Szaflarski, J. P. (2020). Front. Hum. Neurosci. 14:598435. doi: 10.3389/fnhum.2020.598435

In the original article, there was a mistake in Figure $\mathbf{1}$ as published.

The original figure was adapted from Dehkharghani et al. (2015), but this adaptation was not appropriately described and referenced in the manuscript. We apologize for this oversight. The figure has been revised: the adapted portion has been replaced with a new graphic and the caption now appropriately indicates that a portion was adapted from a previously published article. The corrected Figure 1 appears below.

The authors apologize for this error and state that this does not change the scientific conclusions of the article in any way. The original article has been updated.

\section{REFERENCES}

Dehkharghani, S., Mao, H., Howell, L., Zhang, X., Pate, K. S., Magrath, P. R., et al. (2015). Proton resonance frequency chemical shift thermometry: experimental design and validation toward high-resolution noninvasive temperature monitoring and in vivo experience in a non-human primate model of acute ischemic stroke. Am. J. Neuroradiol. 36, 1128-1135. doi: 10.3174/ajnr.A4241

Publisher's Note: All claims expressed in this article are solely those of the authors and do not necessarily represent those of their affiliated organizations, or those of the publisher, the editors and the reviewers. Any product that may be evaluated in this article, or claim that may be made by its manufacturer, is not guaranteed or endorsed by the publisher.

Copyright (C) 2021 Sharma, Nenert, Mueller, Maudsley, Younger and Szaflarski. This is an open-access article distributed under the terms of the Creative Commons Attribution License (CC BY). The use, distribution or reproduction in other forums is permitted, provided the original author(s) and the copyright owner(s) are credited and that the original publication in this journal is cited, in accordance with accepted academic practice. No use, distribution or reproduction is permitted which does not comply with these terms. 

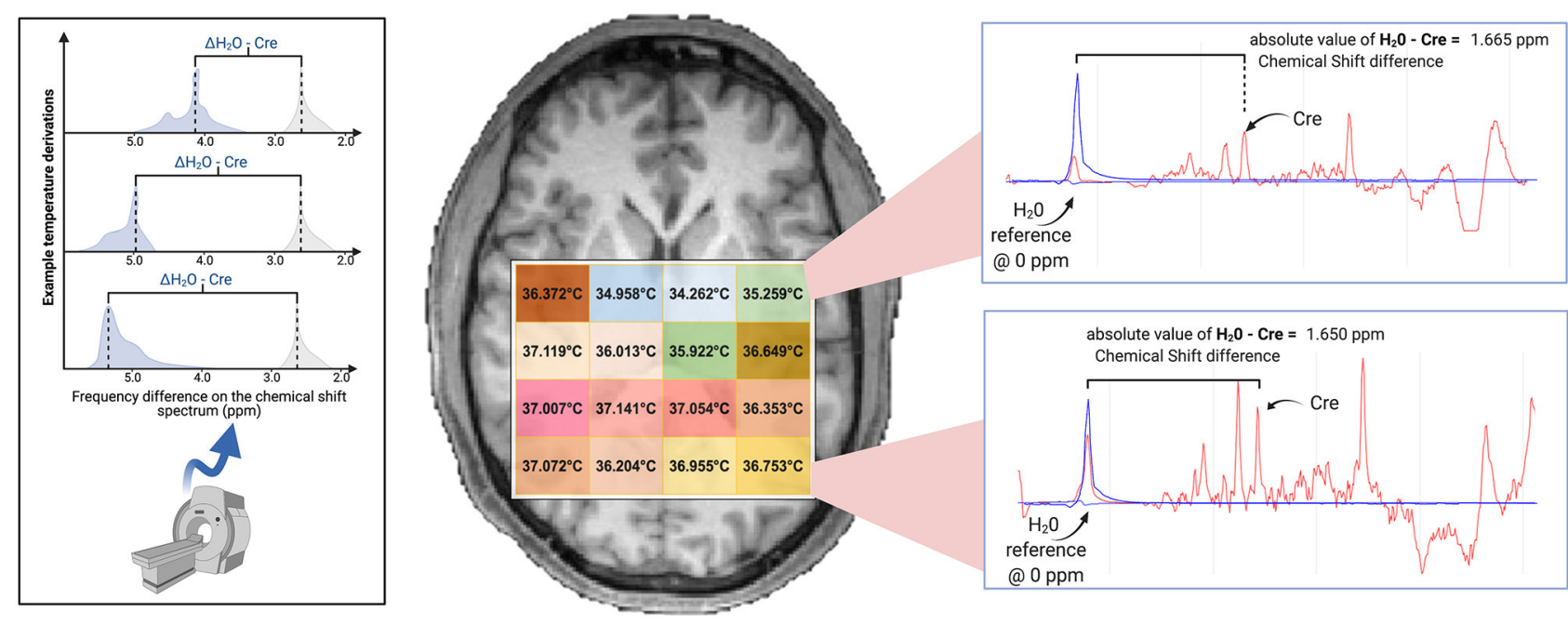

FIGURE 1 | Brain temperature can be non-invasively derived from volumetric magnetic resonance spectroscopic imaging (MRSI) data by calculating the frequency difference between the temperature-sensitive water peak and one or more metabolite peaks that are temperature-insensitive (left $\left.{ }^{*}\right)$. When using creatine as the reference, voxel-level brain temperature can be calculated according to the following equation: $T_{\mathrm{CRE}}=-102.61\left(\Delta_{\mathrm{H} 20-\mathrm{CRE}}\right)+206.1^{\circ} \mathrm{C}, \Delta_{\mathrm{H} 20-\mathrm{CRE}}=\mathrm{chemical}$ shift difference between the creatine and water resonances. Example TCRE calculations are provided for a participant's single tissue slice (right). Representative spectra illustrate $\Delta_{\mathrm{H} 20-\mathrm{CRE}}$ derivations, with plots depicting a water-suppressed metabolite spectrum (red line), with an overlay that indicates the location of the reference water signal (b/ue line). Spectral plots were created within the Metabolite Imaging and Data Analysis System (MIDAS) software package, and the figure was created using BioRender. *Adapted from Dehkharghani et al. (2015). 